\title{
Fatigue damage and sensor development for aircraft structural health monitoring
}

\author{
S.R. Ignatovich ${ }^{\mathrm{a}}$, A. Menou ${ }^{\mathrm{b}}$, M.V. Karuskevich ${ }^{\mathrm{a}}$, P.O. Maruschak ${ }^{\mathrm{c}, *}$ \\ ${ }^{a}$ National Aviation University, Komarova Ave. 1, Kiev 03680, Ukraine \\ ${ }^{\mathrm{b}}$ National Airports Authority of Morocco (ONDA), Academie Mohammed V de l'Aviation Civile (AIAC), Morocco \\ ${ }^{\mathrm{c}}$ Ternopil National Ivan Pul'uj Technical University, Ruska 56, Ternopil 46001, Ukraine
}

\section{A R T I C L E I N F O}

\section{Article history:}

Available online 3 June 2013

\section{Keywords:}

Fracture

Failure

Fatigue damage

Strain localization

Cyclic loading

Deformation relief

\begin{abstract}
A B S T R A C T
It was shown by tests conducted at the National Aviation University (Kiev, Ukraine) that fatigue damage of metal structures may be estimated by sensors with the surface relief pattern to indicate the accumulated fatigue damage. The nature of the deformation relief has been investigated by the light, scan and transmission microscopy, as well as by a new nano-interferometer, developed at the National Aviation University. Fractal analysis of the surface patterns based on box-counting method has proved the efficiency of fractal geometry application for additional quantitative description of such surface structures. The evolution of deformation relief parameters on the sensors surface is determined by the process of the sensor and construction fatigue damage accumulation.
\end{abstract}

(c) 2013 Elsevier Ltd. All rights reserved.

\section{Introduction}

Application of fatigue sensors for Aircraft Structural Health Monitoring (SHM) is aimed on the assessment of structural components fatigue damage at every moment during the aircraft life [1]. The fatigue sensor is a part of the integrated system of sensors, data transmission, and processing ability inside the aircraft structures [2].

Development of new sensors for aircraft structures has been stimulated by very high requirements to the aircraft reliability and strength.

On the first stage of the research and development the single crystal sensors were proposed [3]. It was shown, that the density of the persistent slip bands on the sensor's surface is determined by the accumulated fatigue damage [4].

Now, after investigations of the aluminium alloys under fatigue the efficiency of polycrystalline sensors has been proved $[5,6]$. Appropriate materials for fatigue sensors manufacturing are well known aluminium alloys D16AT, V95, 2024T3, 7075T6, covered by the layer of pure aluminium. This layer acts as an indicator of fatigue damage because the deformation relief appears on the surface under the action of repeated loads [7].

Such sensors look like micro specimens for fatigue tests and are basically the specimen-witness attached to the investigated part of the construction [8]. The possibility to install sensors on the

\footnotetext{
* Corresponding author.

E-mail address: maruschak.tu.edu@gmail.com (P.O. Maruschak).
}

aircraft spar has been considered and correspondent procedure has been proposed.

For polycrystalline sensors the fatigue damage may by estimated by the intensity of deformation relief, i.e., by its dislocation structures on the surface [4]. The surface can be explored by the light microscope with enlargement $200-400 \times$.

For the investigation of deformation relief evolution and correspondent fatigue damage assessment the digital optical system has been developed $[8,9]$. The analysis of the optical digital images of the fatigue sensor surface state gives the data for mathematical models of residual life prediction conducted investigations have shown the efficiency of developed fatigue sensors under wide range of regular and irregular cyclic loading.

\section{Materials and methods}

For a skin of civil aircraft, aluminium alloys D16AT and V95 are widely used in Ukraine and Russia, which are almost analogous to $2024 \mathrm{~T} 3$ and 7075T6. The main alloying components of D16AT and $2024 \mathrm{~T} 3$ are copper and magnesium, while V95 and 7075T6 contain about $5 \%$ of zinc. In order to reduce the possible corrosion process, some sheets of mentioned alloys are often covered with a layer of pure aluminium (for D16AT and 2024T3) or with a layer of Al with $1.0 \%$ of $\mathrm{Zn}$ (for $\mathrm{V95}$ and 7075T6). The thickness of clad layer is ranging from $4 \%$ to $7 \%$ of the total sheet thickness.

For polycrystalline metals as well as for single-crystals, the cyclic loading under certain conditions leads to strain localization zones called persistent slip bands [10,11]. These PSB's are 
connected with the evolution of a dislocation substructure and the formation of extrusion/intrusion on the specimen surface [12].

Aluminium and some of its alloys, which may be used for cladding, are considered to be so called persistent slip bands (PSBs) type materials, because when they are subjected to cyclic loading, PSBs appear and develop on their surfaces. Relief intensity depends on the stress level, distribution of the stress near the stress concentrator and the number of cycles [12,13].

To find appropriate quantitative parameters for the relief description and for the assessment the corresponded accumulated fatigue damage by the surface relief parameters a wide spectrum of systematical tests has been performed $[14,15]$.

Flat specimens with a hole in the center in order to induce fracture localization were used in a presented fatigue test procedure. Such stress concentrator indicates the point for optical investigation as well. The thickness of the specimen is $1.5 \mathrm{~mm}$ and the diameter of the hole is $4.0 \mathrm{~mm}$. These dimensions were chosen because sheets of $1.5 \mathrm{~mm}$ thickness are used in many cases for aircraft skin production, where as $4 \mathrm{~mm}$ hole imitates a constructive hole for rivets. In aircraft structures rivets are used to joint sheets of the skin or mount the skin on frames and stringers. The number of rivets in the structure of a modern passenger airplane for 200 passengers is more than 1.5 million. Thus, such kind of stress concentrator is typical.

Tests have been performed under wide spectrum of loads at frequency of $11 \mathrm{~Hz}$ and load ratio $R=0$.

\section{Surface dislocation structure as an indicator of accumulated fatigue damage}

The procedure of accumulated fatigue damage estimation used in the research includes the analysis of digital images of the deformation relief (Fig. 1) investigated by the light microscope.

Correspondence of the studied structures to the well-known scheme of the extrusion/intrusion formation was proved by the scan microscope investigation [9].

The digital photos of the specimen surface with developed deformation relief obtained by the scan microscope SEM-515 "Phillips" with the voltage $30 \mathrm{kV}$ are presented in Fig. 2.

The dislocation structure of deformation relief was investigated as well. It was revealed that several kinds of dislocation substructures co-exist inside the surface relief: extended dislocation substructure (Fig. 3a), chaotic dislocation in the grain (Fig. 3b),

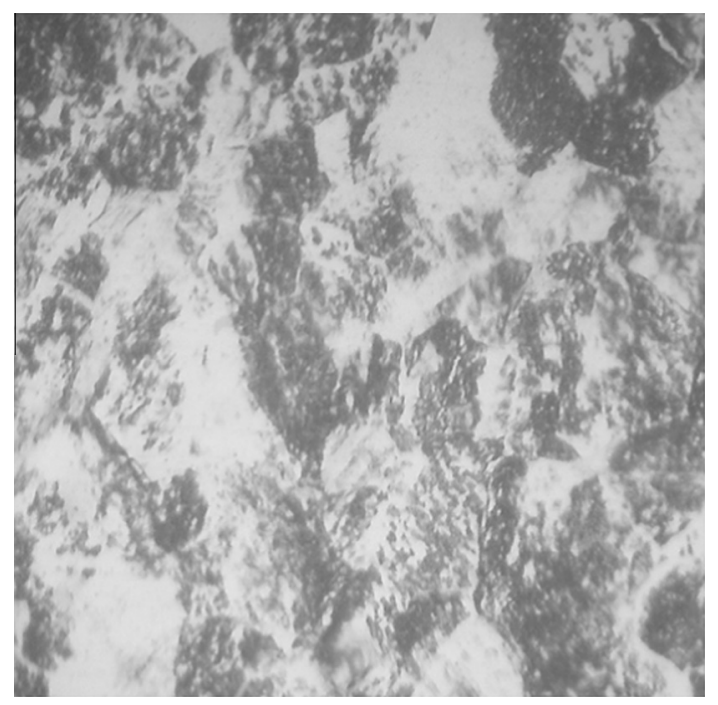

Fig. 1. Optical image of the deformation relief on the alclad aluminium alloy. chaotic dislocations inside the strip-like dislocation (Fig. 3c), subgrain with inner block substructure (Fig. 3d), and honeycomb structure (Fig. 3e).

The images of cyclically loaded specimen surfaces have been processed by special software. The developed program saves the surface images in bmp format and gives the possibility to determine the proposed damage parameter $D$ quantitatively $[14,15]$. Such parameter is estimated near the stress concentrator on the area approximately $0.09 \mathrm{~mm}^{2}$. Damage parameter $D$ is equal to the ratio of the surface area with deformation tracks (PSBs) to the total checked surface in the observed spot.

A set of experimental curves that express the dependence of accumulated damage parameter on the number of cycles has been obtained. Graphs presented in Fig. 4 shows the sensitivity of the surface relief intensity both to number of cycles and stress ratio.

The tests were finished after the nucleation of fatigue crack of $1.0 \mathrm{~mm}$ length, so a crack length of $1.0 \mathrm{~mm}$ has been accepted as the critical state condition.

In practice indication of actual strain level under static loads also is very important; therefore special investigations of surface state under static deformation have been conducted as well. A set of specimens of aluminium alloy D16AT has been tested under maximum relative strain $0.66 \%$; $0.83 \%$; $0.85 \% ; 1.12 \% ; 1.19 \% ; 2.15 \%$; $2.32 \%$; $5.22 \%$; $12.15 \%$ relatively.

Formation and evolution of the deformation relief were observed, but in contrast to fatigue regimes surface pattern have been observed only under the relative strain $2 \%$ and more. So in the case of static loads, deformation relief of Al clad alloys can indicate.

\section{Fractal geometry application for the analysis of intrusion/ extrusion structures}

The search of the additional criteria for deformation relief at quantitative description leads to fractal geometry [14], which is wildly used nowadays at solving the material science problems.

Fractal geometry is a mathematical concept that describes objects of irregular shape. Some natural geometrical shapes, that can be irregular, rough or fragmented, can be described using concepts of fractal geometry as long as the requirement of self-similarity is satisfied. The latter term implies that the geometrical features of an object are independent of the magnification or observation scale [15].

It was supposed that fractal geometry can improve the proposed method of optical diagnostic. The aim of the following test was to justify experimentally the possibility of quantitative estimation of accumulated fatigue damage both by the saturation parameter (damage parameter $D$ ) and fractal dimensions of deformation relief.

Specimens of aluminium alloys D16AT, 2024T3 and 7075T6 have been loaded by bending with the wide range of stresses at frequency $25 \mathrm{~Hz}$. The surface was polished with diamond paste.

Nowadays there are a lot of methods of the fractal dimensions calculation for the nature objects. One of the most widespread is a "box counting" [14]. This method allows to calculate the definite types of fractal dimension.

The first possible type of fractal dimension is fractal dimension of the boundaries of deformation relief spots. This type of fractal dimension is designated as $D p$.

For some fractals the most informative parameter is a fractal dimension of the ratio of perimeter to area. It is known, that this ratio characterizes the shape of objects, and for the regular geometrical figures this parameter is constant value and does not depend on the object size. At the paper [15] this type of fractal dimension was successfully used for the description of the clouds shape. 


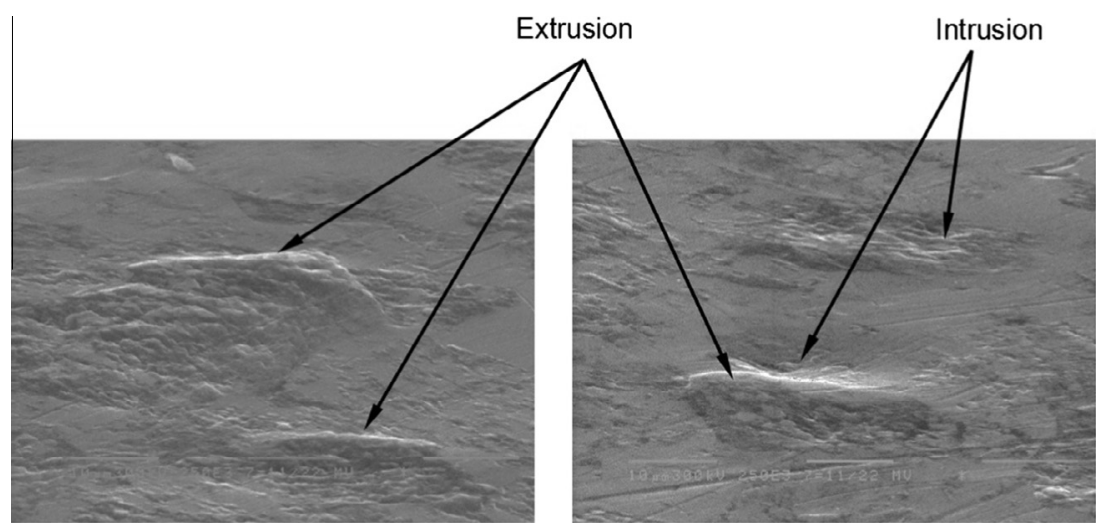

Fig. 2. The fragments of the surface of the aluminium layer of alclad alloy D16AT investigated by scan microscope SEM-515 $(\times 2500)$.
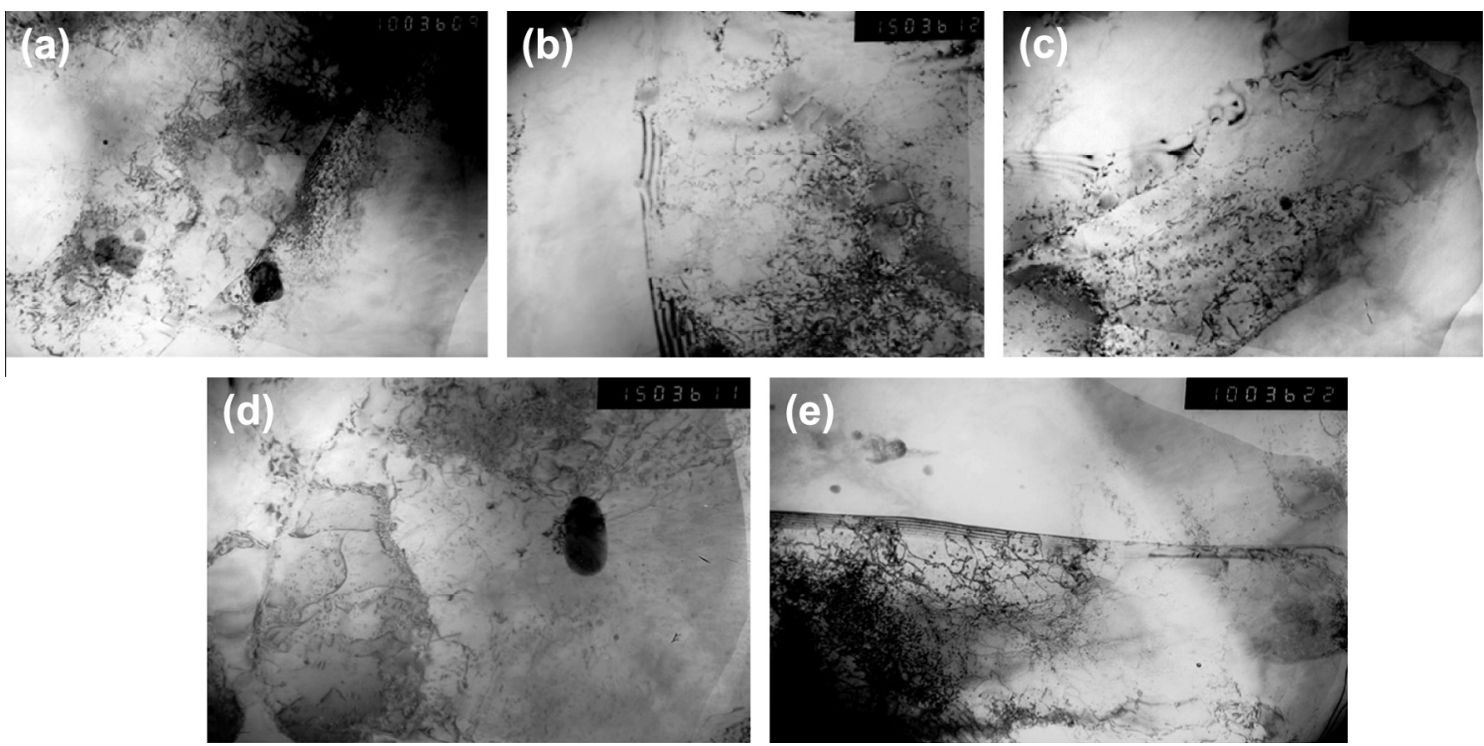

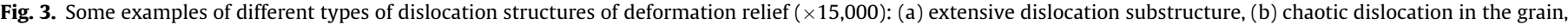
(c) chaotic dislocations inside the strip-like dislocation, (d) sub-grain with inner block substructure, and (e) honeycomb structure [4].

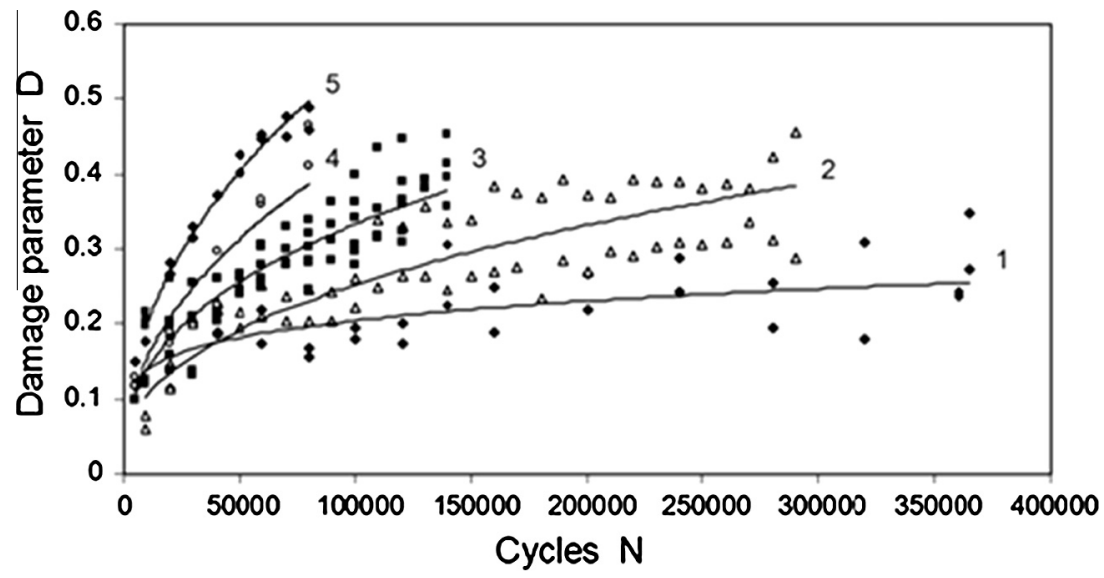

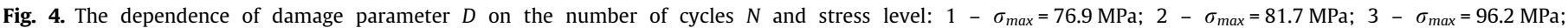
$4-\sigma_{\max }=105.8 \mathrm{MPa} ; 5-\sigma_{\max }=115.4 \mathrm{MPa} ; 6-\sigma_{\max }=129.8 \mathrm{MPa}, 7-\sigma_{\max }=134.6 \mathrm{MPa}$

Correspondent fractal dimension for the clusters of deformation relief will be further called $D p / s$.

For the data processing automation the special software has been developed.
Main stages of calculations are: transformation of digital images of a surface into the monochromic; separation of single clusters of deformation relief; determination of their contours; overlapping of the box net on the cluster's contours or on their surface (Fig. 5a), 
calculation of the box number, overlapping contours of clusters or their surfaces.

Final stage of fractal dimensions calculations is the construction of the corresponding graphs (Fig. 5b) and the determination of the gradients of the relationships: (a) for the fractal dimension of clusters' contours, $\ln N p=f(\ln (1 / \delta))$; (b) for the clusters' surface area, $\ln N s=f(\ln (1 / \delta))$; and (c) for the ratio of perimeter to area, $\ln N p=f(\ln (N s))$, where $N p$ is the number of cells (boxes), overlapping contours of deformation relief clusters; Ns is number of cells overlapping the surface area of deformation relief clusters; $\delta$ is the size of the cell (Fig. 5b).

Fractal dimension $\mathrm{Dp} / \mathrm{s}$ with the application of geometrical method was estimated as a doubled absolute value of the tangent of the slope angle of the middle part of the fractal graph in its linear approximation in log-log coordinates [15].

Fractal dimensions of the deformation relief clusters contours as well as the fractal dimensions determined by the ratio of perimeter to area exceed topological dimension of the line and are within the range of $1-2$.

In the next experiment the specimens were tested under the bending with the maximum cycle stress $173 \mathrm{MPa}$ and minimum stress of the cycle $0 \mathrm{MPa}$. Damage parameter $D$ and fractal dimension $D p / s$ were selected as the main diagnostic parameters. It was presumed that both parameters $D$ and $D p / s$ complement each other. That is why multiple regression models must be used for residual life prediction.

Dispersion and regression analysis of the test data was made with module "ANOVA" of the "Statgraphics Plus". It has shown the possibility of the multiple correlation model application:

$N_{\text {res. }, \%}=180.346-109.588 D-56.6685 D p / s$,

where $D$ is damage parameter; $D p / s$ is fractal dimension; $N_{r e s . \%}$ is the residual number of cycles, \%.

In the obtained model the correspondent value of the $R^{2}$ equals $81.4 \%$ and standard error is 6.48 .

Analysis performed proves the significance of both considered models parameters: damage parameter $D$ and fractal dimension $D p / s$.

\section{Fatigue sensor's conceptual design}

The described above approach to the aluminium fatigue monitoring can be applied for direct diagnostic of structural material state and for the fatigue monitoring by the sensors made of correspondent metal.
The structurally sensitive damage indicator is made of alclad aluminium alloy D16AT. Such choice is caused by the next reasons $[16,17]$ :

- it has been proved the possibility of quantitative estimation of accumulated fatigue damage by the parameters of deformation relief, which is formed on the surface of alclad layer under cyclic loading;

- aluminium alloy D16AT is the basic structural material, that is why it defines phenomenological community with the fracture processes in the sensor and in the most part of structural material of the aircraft.

Conceptual version of the developed sensor presented in Fig. 6 . The distance between holes for the sensor installation is $40.0 \mathrm{~mm}$. The thickness of the sensor $1.2 \mathrm{~mm}$ (see Fig. 7).

Taking into account the wide spectrum of loading condition, it is obvious that a problem of the sensor's sensitivity optimization in accordance with the actual loading of the elements, arises.

In the developed sensor the necessary raising of sensitivity is achieved by the redistribution of stress due to the corresponding distribution of stiffness along the length of sensor. The local stress rise in the test portion of the sensor is defined by the relationship between the width of test portion and the overall dimensions. The test portion of the sensor does not contact with the surface of structural element. The experience of the previous applications of the fatigue damage specimen-witness (fatigue sensors) in aviation was used for the definition the place and the method of the sensor installation. The specimen-witness on airplanes can be placed into the rear spar of the wing. The analysis of modern aircraft structures has shown the advisability of manufacturing basic model of the sensor with the same length between the fixing points. The quality of the sensor surface is reached by the mechanic and electrolytic polishing. It is necessary for the light microscopic analysis of deformation relief, which is formed on the surface of sensor $[3,6]$.

The application of finite element analysis permits to solve a problem of the sensor's geometry optimization for required sensitivity. Among the main stages of simulation is the simulation of team-work of the alclad layer and structural alloy. It is very significant problem because the materials of alclad layer and basic alloy have essentially different mechanical characteristics, particularly proportional limit.

The attachment of the indicator to the specimen for fatigue tests and their team-work under cyclic loading has been also simulated.

In the mentioned tests the sensors were installed on each side of structural components (Fig. 8). Thereby it helps to provide the

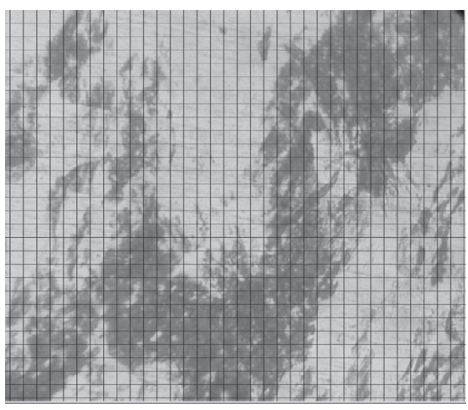

(a)

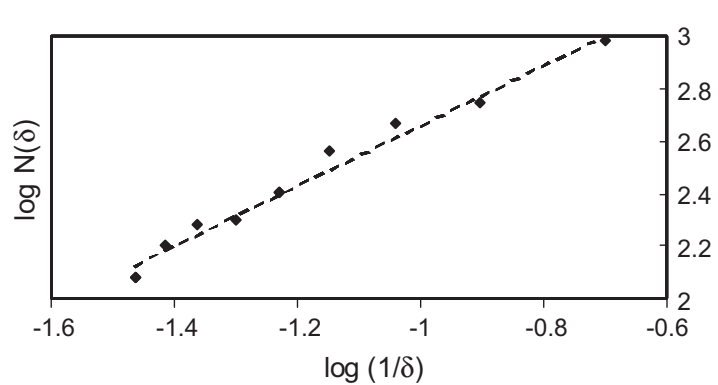

(b)

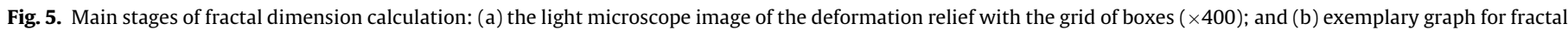

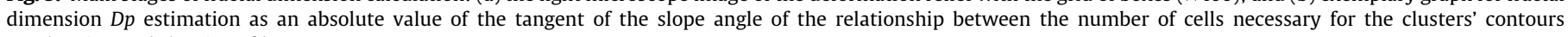
overlapping and the size of boxes. 


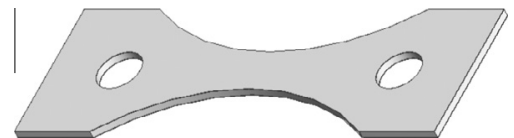

Fig. 6. Conceptual version of the fatigue sensor.

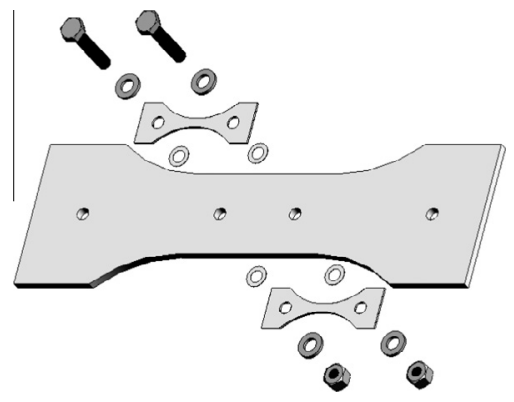

Fig. 7. 3-D image of the sensors attachment to the specimen for fatigue test.

symmetrical loading of the sensors and to receive more information about their damage.

\section{The results of the sensor's surface state monitoring}

The conducted fatigue tests have conformed the ability of the new fatigue sensor application for the fatigue damage monitoring of aviation components. Deformation relief which is formed on the surface of the sensor in the test portion basically is the system of the extrusions, intrusions, persistence slip bands, i.e., the evolution of the sensor surface state is similar to the processes, previously investigated on the surface of alclad aluminium alloys specimens near the stress concentrators $[7,12,13]$.

The deformation relief has been described by damage parameter $D$ and fractal dimensions of deformation relief clusters $D p / s$.

The strong relationship between the selected parameters and number of cycles has been revealed.

Taking into account that the both parameters, namely $D$ and $D p /$ $s$ indicate the accumulated fatigue damage it was proposed to use multiple regression model for the residual life prediction:

$N_{\text {res. }}=A+B \log D+C \log D p / s$,

where $N_{\text {res. }}$ is number of cycles to the fracture; $A, B, C$ are constants; $D$ is damage parameter; and $D p / s$ is fractal dimension.

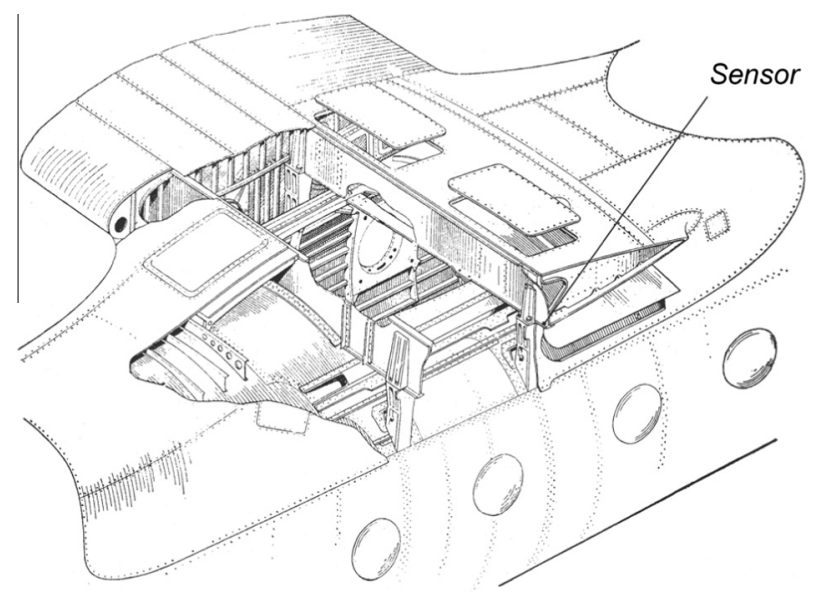

Fig. 8. Area where the fatigue sensor should be installed.
The test conducted under the different loading conditions has shown that the accuracy of mentioned above multiple regression model can be expressed by the coefficient of determination $R^{2}$ in the range from 0.75 to 0.95 .

The practical implementation of new method for fatigue monitoring needs a spectrum of sensor with different sensitivity. The necessary sensitivity can be attained by correspondent geometry of the sensor. The optimization of the sensor geometry can be performed by the application of the Finite Elements Method.

Analysis of the early works on fatigue sensors shows $[3,4,6]$, that for aircraft the place that better fit to the sensor installation is a root section of the aft spar.

\section{Conclusion}

Deformation relief on the surface of alclad aluminium alloys under fluctuating loading can be considered as an indicator of accumulated fatigue damage. The proposed parameters of the deformation relief intensity allow analyzing fatigue sensor's state and correspondent fatigue damage. New fatigue sensor can be used for aircraft structural health monitoring and adopted for some another structures, for example bridges, ships, etc.

\section{References}

[1] M.V. Karuskevich, A.I. Radchenko, E.E. Zasimchuk, Single-crystal as an indicator of fatigue damage, Fatigue and Fracture of Engineering Materials and Structures 15 (1993) 1281-1283.

[2] G. Langfelder, A. Longoni, F. Zaraga, Monitoring fatigue damage growth in polysilicon microstructures under different loading conditions, Sensors and Actuators A: Physical 159 (2) (2010) 233-240.

[3] S.R. Ignatovich, A. Menou, M.V. Karuskevich, P.O. Maruschak, Fatigue sensor for aircraft structural health monitoring, in: Proc. of the International Symposium on Aircraft Materials Advanced Materials: Processing, Properties, Reliability and Diagnostics Fez, Morocco, 2012.

[4] M. Karuskevich, O. Karuskevich, T. Maslak, S. Schepak, Extrusion/intrusion structures as quantitative indicators of accumulated fatigue damage, International Journal of Fatigue 39 (2012) 116-121.

[5] B. Mostefa, A. Abdelkrim, B. Ali, B. Mohamed, Effect of hardening induced by cold expansion on damage fatigue accumulation and life assessment of aluminum alloy 6082 T6, Materials Research 15 (6) (2012) 981-985.

[6] P. Maruschak, I. Konovalenko, M. Karuskevich, V. Gliha, T. Vuherer, Automated diagnostics of damage of aluminum alloy under conditions of high-cycle fatigue, in: Proceedings of the 20th Jubilee Conference on Materials and Technology, Portorož, Slovenia, 2012.

[7] S.V. Panin, A.V. Byakov, V.V. Grenke, I.V. Shakirov, S.A.K. Yussif, Multiscale monitoring of localized plastic strain evolution stages in notched aluminum AA 2024 alloy tension specimens by acoustic emission and television-optical techniques, Physical Mesomechanics 13 (2010) 203-211.

[8] M.V. Karuskevich, E.Yu. Korchuk, A.S. Yakushenko, T. Maslak, Estimation of the accumulated fatigue damage by saturation and fractal dimension of the deformation relief, Strength Mater 40 (2008) 693-698.

[9] S.R. Ignatovich, M.V. Karuskevich, T.P. Maslak, Computer aided optical method for aircraft's components fatigue life estimation, in: Proc. of the International 17-th European Conf. on Fracture Multilevel Approach to Fracture of Materials, Components and Structures, 2-5 September, Brno, Czech Republic, 2008.

[10] P.O. Maruschak, I.M. Zakiev, A.P. Sorochak, O.V. Maruschak, Optical-digital diagnostics of localisation of deformation processes in a steel nanocoated specimen, Strength Fracture and Complexity 7 (4) (2012) 333-339.

[11] V.E. Panin, Yu.V. Grinyaev, V.E. Egorushkin, Foundations of physical mesomechanics of structurally inhomogeneous media, Mechanics of Solids 45 (2010) 501-518.

[12] V.E. Panin, E.E. Deryugin, Mesomechanics of the formation of banded structures on mesoscopic and macroscopic levels, Physics of Metals and Metallography 96 (Suppl.) (2003). S2-S15.

[13] V.E. Panin, Synergetic principles of physical mesomechanics, Theoretical and Applied Fracture Mechanics 37 (2001) 261-298.

[14] J. Feder, Fractals, Plenum Press, New York, 1988.

[15] S. Lovejoy, Area-perimeter relation for rain and cloud areas, Science 216 (1982) 185-187.

[16] G. Lasko, Y. Deryugin, S. Schmauder, Simulation of plastic strain localization in polycrystals, Abstracts for the 14-th US National Congress of Theoretical and Applied Mechanics, Virginia Polytechnic Institute and State University Blacksburg, VA, USA, 2002.

[17] I. Sen, K. Gopinath, R. Datta, U. Ramamurty, Fatigue in Ti-6Al-4V-B alloys, Acta Materialia 58 (2010) 6799-6809. 